\title{
Research on the Model of College Students' Knowledge Development Based on Knowledge Organization Model
}

\author{
He Jie \\ Ningbo Da Hong Ying University \\ Ningbo, China
}

\begin{abstract}
This paper mainly discusses the influence of the knowledge transmission efficiency, the acceptance of organizational knowledge and the proportion of the target group of college students on the organization of individual knowledge of college students. In this paper, a model of college students' knowledge organization in the context of single knowledge and multiple knowledge is established, and the above conclusions are explored through data verification. According to the final model inspired suggestions were put forward for knowledge training mode newly enrolled college students and graduate students of the groups. The innovation of this paper is mainly through the means of models to explore the mass media from the media era of the dissemination of knowledge among students and students to build their own knowledge.
\end{abstract}

Keywords—knowledge organization; groups of college students; knowledge training model; mobile media dissemination

\section{INTRODUCTION}

The classical knowledge transmission mode mainly includes linear model, circular pattern and systematic pattern, For example: Lasswell "5W" model, Osgood - Schramm model and Bello model. With the advent of the information age, intelligent mobile terminal has become the main channel for students to acquire knowledge, the university students' knowledge training mode has undergone great changes ${ }^{[1,2]}$, Such as Web2.0 under the Blog, Tag, SNS, RSS, Wiki, Pod casting, Social Bookmarking, IM, Mash-up and other carrier applications. And then, the mode of transmission of knowledge and training mode of college students has changed.

The efficiency of knowledge acquisition based on individual and group knowledge differences of college students depends not only on the number of students' knowledge matching with their corresponding groups, but also on the efficient dissemination of knowledge among students in order to achieve the individual knowledge organization of students ${ }^{[3]}$ In the process of organization of students' personal knowledge, there are many factors that impede the smooth transition of knowledge to organizational knowledge. Among these constraints, the student's personal factors are the most important ${ }^{[4]}$. Previous studies have shown that personal knowledge organization in the process of personal factors mainly in the following two aspects ${ }^{[5]}$. The first is the tendency of personal knowledge sharing, the second is the cumulative accumulation of personal knowledge.
Personal knowledge sharing tendency refers to the student group, students will own knowledge through the hands of the mobile terminal to publish relevant information, and unconditionally impart to others wishes. Usually students in these groups spread knowledge to other students if they can get praise, comments or other returns, the knowledge sharing tendency is more intense ${ }^{[6]}$. Zhang Lingli carried out the goaloriented network-based knowledge dissemination experiment between students, not only to improve students 'listening ability and English comprehensive application ability, but also to stimulate students' enthusiasm for learning and cultivate their self-learning ability ${ }^{[7]}$. In the mode of communication between students, there are early Web2.0 model of knowledge transmission, Web2.0 also known as "read and write network", "participatory network" or "people-centered network" mode stage ${ }^{[8]}$. Zhang Xuebo etc. From the Web2.0 era of micro-blog education application practice, the micro-blog and so on in the dissemination of the dissemination of the effect of communication and dissemination mode reveals the spread of micro-blog on the traditional mass communication theory impact $^{[9]}$.

This paper tries to construct the individual knowledge organization model of college students under the influence of various knowledge, and on the basis of the model analysis, through the numerical test, the quantitative study of individual knowledge organization degree, explores the core population groups of students own knowledge degree of organization spread through convenient mobile terminal to its student population as the center of the main propagation efficiency.

\section{MODEL CONSTRUCTION}

The individual knowledge organization of individual students is realized through the knowledge transmission among the groups of their circle of friends. According to this, we think that the degree of students' individual knowledge organization can be controlled by the students who master certain knowledge in the organization; therefore, in a single knowledge and a variety of knowledge of these two conditions, we were to establish the knowledge of individual student organization model $^{[10]}$. 


\section{A. Single knowledge}

Suppose a group of students to master some new knowledge of students as the core students, not master new knowledge $X$, the students that need for core students to teach as the target. The knowledge transfer between the core students and the target students is a one to many mapping relationship. That is a core student can simultaneously to multiple targets students in the dissemination of knowledge, but a student will only accept a core goal to teach students knowledge. Suppose each core within the unit time to teach students new knowledge $X$ to the target number is $\lambda$. Since the process only teach new knowledge $X$, Target students do not have the opportunity to choose other knowledge, New knowledge $X$ has the highest transmission efficiency at this time.

For a student group, suppose the total number of students is $\mathrm{N}$, and the ratio of the core student to the target student is the function $\alpha(t)$ and $\beta(t)$. Since the relationship between $\alpha(t)$ and $\beta(t)$ is $\alpha(t)+\beta(t)=1$. From the foregoing assumptions, In the period $\Delta t$, the number of core students added by the organization is::

$$
N(\alpha(\Delta t+t)-\alpha(t))=\lambda N \alpha(t) \beta(t) \Delta t
$$

Then, students' personal knowledge organization model can be obtained under the condition of knowledge is:

$$
\frac{d \alpha(t)}{d t}=\lambda \alpha(t) \beta(t)
$$

While $\alpha(t)+\beta(t)=1$, if the initial state, the organization's core students accounted for the proportion of the total number of students is $\alpha_{0}$. Substituting it into (2), can be solved:

$$
\alpha(t)=\frac{1}{1+\left(\alpha_{0}^{-1}-1\right) e^{-\lambda t}}
$$

As $d \alpha(t) / d t=\lambda \alpha(t) \beta(t)=\lambda \alpha(t)(1-\alpha(t))=-\lambda(\alpha(t)-1 / 2)^{2}+\lambda / 4$, Therefore, when the proportion of core students within the organization to half the total number of students within the organization, new knowledge $X$ propagation within the tissue reaches a maximum is $\lambda / 4$. By the formula (3) shows that, while $t \rightarrow+\infty, \alpha(t)$ will tend to 1 , That is as long as the time long enough, corresponding to all students in the student group will master this new knowledge, and then to become the core students. Therefore, When students within the organization can only choose new knowledge $X$, new knowledge $X$ space overflow process shown in Fig. 1:

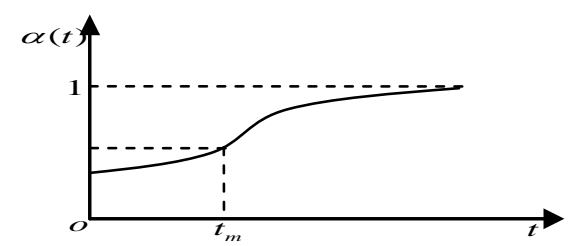

Fig. 1. The proportion of core students in a single knowledge situation
As can be seen from Fig. 1-1, when a student group organization only a new knowledge of the case, after a sufficiently long period of time, the degree of organization of students' personal knowledge will reach a very high level. But in reality, it is impossible for only one kind of knowledge to be spread in the group. Therefore, it is an ideal process of individual knowledge organization.

\section{B. More than two kinds of knowledge}

Suppose that a student in a group organization, there are a variety of knowledge, and the mutual exclusion of knowledge between the characteristics of the organization's students only choose to accept the most suitable for their own situation of a knowledge. We will need to organize the knowledge expressed as $\boldsymbol{X}$, other knowledge is expressed as $y_{i}(\boldsymbol{i}=1,2,3, \ldots \boldsymbol{i}$ Different values represent different knowledge), The total number of students in the organization is $N$. Since other knowledge is $y_{i}$, after some of the target students received knowledge $X$ from the core students, According to their interests, hobbies, such as the direction of time scheduling and other different, Eventually giving up knowledge $X$ instead to choose more suited to their own situation of knowledge $y_{i}$, We called the students giving up new knowledge new students. If the proportion of core students, target students and new students ratio of the total number of students are $\alpha(t), \beta(t)$ and $\gamma(t)$, Then ,we can get $\alpha(t)+\beta(t)+\gamma(t)=1$. Assuming the organization of students' personal knowledge, the proportion of new students to new core students is $\mu$, then, we can get:

$$
N(\alpha(t+\Delta t)-\alpha(\Delta t))=\lambda N \alpha(t) \beta(t) \Delta t-\mu N \alpha(t) \Delta t
$$

Formula (4) can be further simplified as:

$$
\frac{d \alpha(t)}{d t}=\lambda \alpha(t) \beta(t)-\mu \alpha(t)
$$

For new students group:

$$
\frac{d \gamma(t)}{d t}=\mu \alpha(t)
$$

Suppose the initial stage, the organization's core students and target students accounted for the proportion of the total number of students were $\alpha_{0}$ and $\beta_{0}$, Combined with the formula (5) and (6), And taking $\alpha(t)+\beta(t)+\gamma(t)=1$ into account, the model of personal knowledge organization under the condition of many kinds of knowledge is:

$$
\left\{\begin{array}{l}
\frac{d \alpha(t)}{d t}=\lambda \alpha(t) \beta(t)-\mu \alpha(t) \\
\frac{d \gamma(t)}{d t}=\mu \alpha(t) \\
\alpha(t)+\beta(t)+\gamma(t)=1 \\
\alpha(0)=\alpha_{0}, \beta(0)=\beta_{0}
\end{array}\right.
$$


In the model (7), For $\alpha(t)+\beta(t)+\gamma(t)=1$ derivative, there are:

$$
\frac{d \alpha(t)}{d t}+\frac{d \beta(t)}{d t}+\frac{d \gamma(t)}{d t}=0
$$

Substituting equation (5) and formula (6) into formula (8), we get:

$$
\frac{d \beta(t)}{d t}=-\lambda \alpha(t) \beta(t)
$$

As a result of $\alpha(0)=\alpha_{0}$, it is substituted into formula (9), and combined with formula (6) solution:

$$
\beta(t)=\alpha_{0} e^{-\frac{\lambda}{\mu} \gamma(t)}
$$

Substituting equation (9) into formula (5) we can get:

$$
\frac{d \alpha(t)}{d t}=-\frac{d \beta(t)}{d t}+\frac{\mu}{\lambda \beta(t)} \frac{d \beta(t)}{d t}
$$

The formula (11) treated as follows:

$$
\frac{d \alpha(t)}{d t}=-\frac{d \beta(t)}{d t}+\frac{\mu}{\lambda \beta(t)} \frac{d \beta(t)}{d t}=\frac{d \beta(t)}{d t}\left(\frac{\mu}{\lambda \beta(t)}-1\right)
$$

Due to the number of students in the target tissue over time and gradually reduced, $\beta(t)$ must be a decreasing function, That is $\beta^{\prime}(t)<0$, When the knowledge $X$ began to spread among students, the target students accounted for the largest proportion of the total number of students: $\beta_{\max }=\beta_{0}$. As can be seen from (12), while $\mu / \lambda<\beta_{0}, \alpha^{\prime}(t)>0$, At this point $\alpha(t)$ is an increasing function; While $\mu / \lambda>\beta_{0}$, $\alpha^{\prime}(t)<0$, At this time $\alpha(t)$ is a decreasing function. Therefore, the degree of organization of students' individual knowledge is related to the values of $\lambda, \mu$ and $\beta_{0}$.

While $\mu / \lambda>\beta_{0}, \alpha(t)$ is a decreasing function, then $\alpha_{\max }=\alpha_{0}$, That is the core of the initial stage of the number of students is the maximum number of students in the core of the process of dissemination of knowledge $X$. This indicates that, in this case, knowledge $X$ has not spread within the student population; On the contrary, the original master of knowledge $X$ core students over time also gradually give up knowledge $X$, and then choose a more appropriate knowledge $y_{i}$. After a sufficiently long period of time, $\alpha(t)$ tend to 0 , means that knowledge of the organization $X$ was eventually eliminated.

While $\mu / \lambda<\beta_{0}, \alpha(t)$ is an increasing function, Over time, the inner core of the organization of students is increasing, and the target students continues to decline. When the target student is reduced to the proportion of the total number of students is $\beta\left(t_{m}\right)$, and $\beta\left(t_{m}\right)$ satisfying $\beta\left(t_{m}\right)=\mu / \lambda$, $\alpha(t)$ reaches the maximum value $\alpha\left(t_{m}\right)$. After that, $\alpha(t)$ will again decrease gradually, And finally tend to zero. In this process, the number of core students in the organization increased first and then decreased, indicating that in a certain period of knowledge $X$ dissemination, it contains certain groups of students in a number of students to receive knowledge $X$, The degree of organization of knowledge $X$ can be expressed by the ratio of the core students to the total number of students in the organization.

From the above two cases we can see, regardless of what value $\boldsymbol{\lambda}, \boldsymbol{\mu}$ and $\boldsymbol{\beta}_{\mathrm{o}}$, After a long enough time, the core students will disappear, indicating that all students in the organization have finally given up the knowledge $X$, this is in line with the reality of the actual organization. Because in the era of knowledge economy, a variety of new knowledge emerging, the organization in order to maintain the vitality and competitiveness, must continue to eliminate obsolete knowledge, and create and accept new knowledge.

While $\alpha_{\infty}=0$, there is $\beta_{\infty}+\gamma_{\infty}=1$, according to (110 ), we can get $\beta_{\infty}=\alpha_{0} e^{-\lambda \gamma_{\infty} / \mu}$, so, while $\alpha_{\infty}=0, \beta(t)$ will also be stable at $\beta_{\infty}$, and $\beta_{\infty}$ satisfying $\beta_{\infty} \in[0, \mu / \lambda]$. If $\beta(t)$ and $\alpha(t)$ represents the abscissa and ordinate, Then the relationship between $\alpha(t)$ and $\beta(t)$ can be represented by Fig. 2:

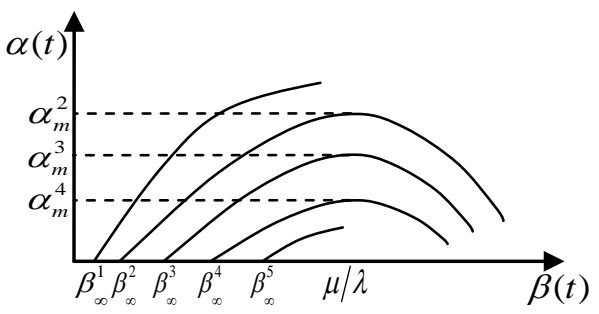

Note $_{\alpha_{m}, i=1,2,3 \ldots .}$ The proportion of different groups of knowledge to accept $\beta_{\infty}^{i}, i=1,2,3 \ldots$... Represent different knowledge to accept project team proportions

Fig. 2. Change in the ratio of core students to target students under various knowledge conditions

\section{NUMERICAL VALIDATION}

Since $\alpha(0)=\alpha_{0}, \beta(0)=\beta_{0}$, equation (11) on both sides of the integral operation can be obtained:

$$
\alpha(t)=\left(\alpha_{0}+\beta_{0}\right)-\beta(t)+\frac{\mu}{\lambda} \ln \frac{\beta(t)}{\beta_{0}}
$$


From the previous analysis, we can see that any kind of organizational knowledge, after a long enough time, will eventually be eliminated. We can substitute $\alpha_{\infty}=0$ into (13), can get:

$$
\left(\alpha_{0}+\beta_{0}\right)-\beta_{\infty}+\frac{\mu}{\lambda} \ln \frac{\beta(t)}{\beta_{0}}=0
$$

While $\frac{\mu}{\lambda}<\beta_{0}$, The maximum value of $\alpha(t)$ is:

$$
\alpha_{\mathrm{m}}=\alpha_{0}+\beta_{0}-\frac{\mu}{\lambda}\left(1+\ln \frac{\mu}{\lambda \beta_{0}}\right)
$$

If there are two organizations $\mathrm{A}$ and $\mathrm{B}$ knowledge of students at the same time the process of individual knowledge organization. In the initial state, The proportion of the core student and the target student in the total number of students in the organization $\mathrm{A}$ is $\alpha_{0}{ }^{\mathrm{A}}=0.05$ and $\beta_{0}{ }^{\mathrm{A}}=0.95$ respectively; The ratio of the core student to the target student in the total number of students in the organization $\mathrm{B}$ is $\alpha_{0}{ }^{B}=0.25$ and $\beta_{0}{ }^{B}=0.75$. Then when $\lambda$ and $\mu$ are different values, According to (14) and formula (15), using Matlab7.0 software calculation, we can get the value of $\alpha_{m}^{\mathrm{A}}, \beta_{\infty}^{\mathrm{A}}, \alpha_{\infty}^{\mathrm{B}}, \beta_{m}^{B}, \gamma_{\infty}^{\mathrm{A}}$ and $\gamma_{\infty}^{B}$ as shown in Table I and Table II:

TABLE I. ORGANIZATION OF STUDENTS IN THE ORGANIZATION OF PERSONAL KNOWLEDGE DEGREE

\begin{tabular}{|c|c|c|c|c|c|c|}
\hline$\lambda$ & $\mu$ & $\alpha_{0}^{\mathrm{A}}$ & $\beta_{0}^{\mathrm{A}}$ & $\alpha_{m}^{\mathrm{A}}$ & $\beta_{\infty}^{\mathrm{A}}$ & $\gamma_{\infty}^{\mathrm{A}}$ \\
\hline 2 & 0.79 & 0.06 & 0.94 & 0.90 & 0.15 & 0.85 \\
\hline 1 & 0.79 & 0.06 & 0.94 & 0.15 & 0.59 & 0.41 \\
\hline 1 & 0.78 & 0.06 & 0.94 & 0.33 & 0.56 & 0.44 \\
\hline 0.8 & 0.78 & 0.06 & 0.94 & 0.05 & 0.69 & 0.31 \\
\hline 0.6 & 0.67 & 0.06 & 0.94 & 0.05 & 0.80 & 0.20 \\
\hline
\end{tabular}

TABLE II. ORGANIZATION OF STUDENTS IN THE ORGANIZATION OF PERSONAL KNOWLEDGE DEGREE

\begin{tabular}{|c|c|c|c|c|c|c|}
\hline$\lambda$ & $\mu$ & $\alpha_{0}^{B}$ & $\beta_{0}^{B}$ & $\alpha_{m}^{B}$ & $\beta_{\infty}^{B}$ & $\gamma_{\infty}^{B}$ \\
\hline 2 & 0.9 & 0.28 & 0.72 & 0.78 & 0.19 & 0.81 \\
\hline 1 & 0.6 & 0.28 & 0.72 & 0.65 & 0.24 & 0.76 \\
\hline 1 & 0.6 & 0.28 & 0.72 & 0.55 & 0.22 & 0.78 \\
\hline 0.8 & 0.5 & 0.28 & 0.72 & 0.25 & 0.21 & 0.79 \\
\hline 0.8 & 0.4 & 0.28 & 0.72 & 0.25 & 0.13 & 0.87 \\
\hline
\end{tabular}

Through the analysis of Table 1 and Table 2, we can find:

a. While $\mu / \lambda<\beta_{0}$, and the values of $\lambda$ and $\mu$ in the tissue $\mathrm{A}$ are the same, we can get $\alpha_{m}^{\mathrm{A}}>\alpha_{m}^{\mathrm{B}}$. Therefore, in contrast to organization $B$, the degree of organization of knowledge $X$ in organization $\mathrm{A}$ is high. Therefore, while $\mu / \lambda<\beta_{0}, \lambda$ and $\mu$ unchanged, if the target of an organization more students, the knowledge of the students the higher the degree of personal knowledge organization.

b. While $\mu$ unchanged, $\lambda$ increase, $\gamma_{\infty}^{\mathrm{A}}$ and $\gamma_{\infty}^{B}$ also increased, that is, the proportion of new students within the organization increased. Analysis of its causes, $\lambda$ increase is the essence of the dissemination of knowledge $X$ among students to improve efficiency. Increasing the efficiency of knowledge $X$ communication leads to more target students in the organization receiving knowledge $X$, The target student who received the knowledge $X$ will compare it with other knowledge in the organization, analyze and select the most suitable level. Once the other knowledge is selected, they will abandon the knowledge $X$ and become the new student.

c. While $\lambda$ unchanged, $\mu$ increase, $\gamma_{\infty}^{\mathrm{A}}$ and $\gamma_{\infty}^{B}$ decrease, that is the proportion of new students within the organization decreased. Analysis of its causes, the increase of $\mu$, which means that the proportion of students who choose to give up after receiving knowledge $X$ in the organization increases in the core students. In this case, knowledge $X$ in the organization of the spread of students between the efficiency of decline, resulting in many target students can't effectively receive the knowledge $X$, and thus lack of comparison with other knowledge within the organization object, reducing the organization of students to choose their own level, and become a new type of student opportunities.

\section{CONCLUSIONS AND IMPLICATIONS}

In the context of a large number of Internet mobile terminal applications, in order to improve the effect and efficiency of students 'knowledge, it is necessary to enlarge the proportion of new students in the organization while trying to improve the degree of students' knowledge organization. From the above analysis, we can see that the degree of organization of personal knowledge of students and the dissemination of knowledge among students, the acceptance of organizational knowledge and the proportion of target students. There are three ways to improve the degree of personal knowledge organization: Improve the efficiency of knowledge dissemination among the students, pay attention to guide various types of mobile terminals and mobile applications students between; Enhance students' organizational knowledge acceptance, encourage more students to make good use of mobile terminals for multichannel self-learning; Expand the proportion of the target students in the organization, make good use of student cadres, graduating class students through convenient mobile terminals and new students in the exchange of knowledge and communication. Based on the above findings, we have the following management implications:

First, the newly enrolled students, whose college life, knowledge and learning is still in the exploratory stage, the organizational structure has not yet fully formed; Their interest is more variable, resulting in low efficiency of knowledge 
transmission between them, and a specific community organizations, the collective sense of honor and the sense of team is relatively weak, making its acceptance of organizational knowledge is not high. Therefore, on the one hand, the university administrators at this stage should strengthen the communication and communication among the students, cultivate the backbone students, construct the learning organization, and improve the communication efficiency of the guidance knowledge among the students. On the other hand, universities should strengthen the organization to promote its core values, strengthen the collective consciousness of students to establish the concept of team, to improve students' knowledge of organizational acceptance (Kozlowski, 2006).

Second, high-grade college students, whose university study, life is relatively stable, Students in the organization usually have chosen to fit their own actual situation, but also to meet the basic requirements of the university's knowledge of university graduation, resulting in the proportion of students within the target low.

On the basis of theoretical research, this paper constructs a model of individual knowledge organization under the condition of single knowledge and multiple knowledge. The study found that the degree of organization of students' knowledge of personal knowledge under a single, although very high, but this is only an idealized state. While the process of individual knowledge organization of students under a variety of knowledge is more complicated, But through the model analysis, combined with numerical tests demonstrate the knowledge dissemination efficiency, organizational knowledge and the proportion of target students to accept the proportion of factors such as the degree of organization of personal knowledge, the results of the actual situation of the organization is consistent. The results of this paper can provide a reference for university students' work management personnel to combine the single individual in the era of mobile Internet terminal is a media transmission point for the different stages of college students to provide knowledge and training mode of decision-making reference.

\section{REFERENCES}

[1] Zhenger Y, Zhikui L. Research on Vertical Search Engine System of Learning Resources Based on Mobile Terminal [J]. Modern Computer: Popularization, 2013 (1): 22-25. (In Chinese)

[2] Dejun Y, Lirong Z,etc. Discussion on the Experiment of Change of Students' Learning Methods Based on Hand - held Mobile Terminal [J] .New Course Teaching: Electronic Edition, 2014 (5): 12-12.

[3] Shao D, Design and Application of Microtrading Based on Learning of Mobile Terminal - Taking "Photoshop" as an Example [J] .S snow Lotus, 2015 (29).

[4] Commings J.Knowledge Sharing:A Review of Literature[R].The Word Bank Operations Evaluation Department,2003:1-57

[5] Claudio Garavelli,Michele Gorgoglione,Barbara Scozzi.Manag-ing Knowledge Transfer by Knowledge Technologies[J]. Tech-novation, 2002(22): 269- 279

[6] Hussar P, Tokin I, Hussar U, et al. Application of Photoshop-based image analysis and TUNEL for the distribution and quantification of dexamethasone- induced apoptotic cells in rat thymus.[J]. Medicina, 2006, 42(6): 504- 12.

[7] Ling-li ZH. Study on Autonomous Learning Model of Web-based English Listening Based on Goal Orientation [J] .Approach of Foreign Languages, 2009, (9): 46. (In Chinese)

[8] Geping L. Qian L. E-Learning 2.0 [J]. Audio-Visual Education Research, 2009, (2): 27.

[9] Xiang Z, Chen Y. Dissemination model and impact evaluation of rumor in microblog[J]. Science Research Management, 2016.

[10] Tianying J, Cong Ch. Analysis on a Model of Individual Staff's Knowledge Systematization[J]. Journal of Intelligence, 2010, 29 (5): 1215. (In Chinese) 